\title{
A rare case of Meckel's diverticulum perforation caused by a toothpick mimicking acute appendicitis
}

\author{
Aleksandar Resanovic ${ }^{1}$, Milan Gojgic ${ }^{1}$, Vladimir Resanovic ${ }^{2}$, Mazen Arafeh ${ }^{3}$ \\ ${ }^{1}$ Department of Surgery, University Hospital Bezanijska Kosa, Belgrade, Serbia \\ ${ }^{2}$ Emergency center, Clinic for urgent surgery, Clinical Center Of Serbia, Belgrade, Serbia \\ ${ }^{3}$ Cocoona Center for aesthetic transformation, Dubai, UAE
}

\begin{abstract}
Meckel's diverticulum (MD) is the most common congenital abnormality of the gastrointestinal tract and is caused by the failure of omphalomesenteric duct to obliterate during embryonal maturation. Most patients are asymptomatic with only 4-16\% presenting complications which include hemorrhage, intestinal obstruction, inflamation and occasionally perforation. A preoperative diagnosis of a complicated Meckel's diverticulum may be challenging because of the overlapping clinical and imaging features of other acute surgical and inflammatory conditions of the abdomen. A 32-year old male was admitted to the emergency department with a 48-hour history of colicky like pain in the lower part of the abdomen, which was gradually increased and was localised in the lower right quadrant, with no history of fever, nausea or vommiting, no urinary symptoms nor altered bowel habits. Laboratory revealed leukocytosis and elevated CRP, while on physical examination guarding and rebound tenderness were found in the right lower quadrant. Abdominal ultrasound established a distended small bowel with very slow peristalsis in ileocecal region. Complete workup raised suspicion for an acute appendicitis, therefore the patient was operated. Intraoperatively, the perforated Meckel's diveriticulum was found, caused by a toothpick. Diverticulectomy and appendectomy were done. Postoperative course was uneventful. Regardless of the fact that a variety of diagnostic procedures are at the disposal of surgeons, we feel that the diagnose of a complicated MD remains a challenging task, largely in thanks to the fact that it mimics some other acute abdominal pathology and, often, is misdiagnosed.
\end{abstract}

Keywords: Meckel diverticula perforation; acute appendicitis

\section{Introduction}

Meckel's diverticulum (MD) is the most common congenital abnormality of the gastrointestinal tract and is caused by the failure of omphalomesenteric duct to obliterate during embryonal maturation. It is a true diverticulum, containing all three layers of intestinal wall, and is located on the anti-mesenteric border. ${ }^{[1]}$ Despite the fact that this condition is common abnormality of the gastrointestinal (GI) tract, most patients are asymptomatic with only 4-16\% presenting complications which include hemorrhage, intestinal obstruction, inflamation, and occasionally perforation. ${ }^{[2]}$ Complications are much more common in males, and the incidence of complications decreases with age, with the majority occuring in pediatric population. Most common clinical presentation of a complicated MD in children is hemorrhage. In adults it is intestional obstruction, then inflammatory process and, less comon, perforation. ${ }^{[3]}$ The diagnosis

\footnotetext{
Copyright (C) 2018 Aleksandar Resanovic et al.

doi: $10.18686 /$ aem.v7i1.119

This is an open-access article distributed under the terms of the Creative Commons Attribution Unported License

(http://creativecommons.org/licenses/by-nc/4.0/), which permits unrestricted use, distribution, and reproduction in any medium, provided the original work is properly cited.
} 
of complicated MD is difficult because of its various presentations which, in some cases, presents as an acute appendictis which is among the most common causes of acute abdominal pain and for emergency surgery. ${ }^{[4]}$

\section{Case report}

A 32-year old male was admitted to the emergency department with a 48-hour history of colicky like abdominal pain in lower part of abdomen, which was gradually increased and localising in the lower right quadrant, with no history of fever, nausea or vommiting, no urinary symptoms nor altered bowel habits.

In medical records, the patient was cleared for allergies and prior surgery. The only thing tah stood out was the fact that he had been using testosteron depo for weight-lifting and gaining muscle.

On addmision, body habitus was mesomorphic (muscular and athletic), physical examination showed guarding and tenderness in the right illiac fossa (Rovsing sign) with positive rebound tenderness (Blumberg sign), axillary temperature of $36,8{ }^{\circ} \mathrm{C}$, rectal temperature of $38{ }^{\circ} \mathrm{C}$, and elevated inflammatory markers - leucocyte level of $17.9 \times 10^{9} / \mathrm{L}(81 \%$ of neutrophiles) and $\mathrm{C}$ - reactive protein level of $104.1 \mathrm{mg} / \mathrm{L}$. Urin analysis was normal. Erect chest and abdomen $\mathrm{X}$ rays were also normal, echosonography of the abdomen showed distended small bowel with very slow peristalsis in ileocecal region with no visuelisation of the appendix, no free fluid in the abdomen and other findings on the abdominal viscera were normal.

Complete physical, laboratory and radiology workup raised suspicion for an acute appendicitis, therefore, it was decided that a surgical intervention should be performed. The patient was consented and taken to theatre. Upon exploration of the abdominal cavity, purulent fluid was found in the ileocecal region, but with no signs of appendicitis, therefore the terminal ileum was further explored. Approximately, $50 \mathrm{~cm}$ the ileocecal valve a perforated Meckel's diverticulum was found, with a penetrating wooden, sharp foreing body (part of the toothpick) ( figure 1 ). Considering these findings, Meckel's diverticulum was exised with a two-layer small bowel suture, and classical appendectomy, followed by drainage of the abdominal cavity. Postoperative course was uneventful. Gradually, oral intake was established with regular digestive function. Drain was extracted on the 2nd postoperativne day, patient was discharged on the 4th postoperative day.

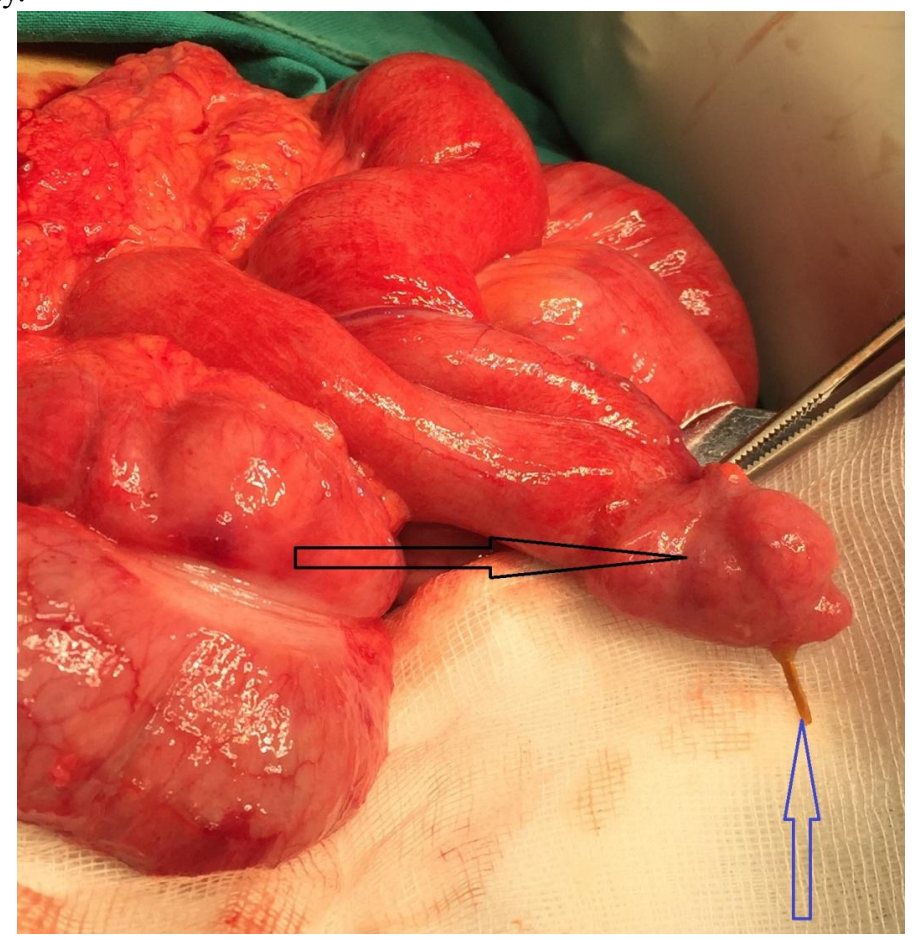

Figure 1; Black arrow is pointing towards the Meckel diverticlum, while the blue arrow is indicating a foreign body perforation ( toothpick ).

On follow-up examination of 10 days and 3 months after surgery, he was doing well. 


\section{Discussion}

Meckel's diverticulum is a congenital, intestinal blindpouch that results from an incomplete obliteration of the vitelline duct. Wilhelm Fabricius Hildanus, a German surgeon, first describedthe diverticulum in 1598. However, the entity was not named until 1809, when Johann Friedrich Meckelthe Younger first reported his research on the diverticulum'sanatomy and embryology. Furthermore, Meckel showed that incomplete obliteration of the vitelline duct results in not only Meckel's diverticulum but also enterocysts, intestinal-umbilical fistulas, and mesodiverticular bands. ${ }^{[5]} \mathrm{MD}$ is the most prevalent congenital anomaly of the gastrointestinal tract, affecting approximately $2 \%$ of the general population. A 3:2 male to female ratio has been reported. MD is true diverticulum because its wall contains all of the layers found in normal intestine. Their location varies among individual patients, but they are usually found in the ileum within $100 \mathrm{~cm}$ of the ileocecal valve. ${ }^{[6]} \mathrm{MD}$ is asymptomatic in most of cases and only about 4 to $16 \%$, and, in some studies, up to $25 \%$ of cases develop complications. However, it can present with a picture of intestinal bleeding in younger age groups or intestinal obstruction, inflammation and perforation in adults. ${ }^{[7]}$

Perforation of Meckel's diverticulum by a foreign body represents a rare complication since the majority of the ingested objects pass through the gastrointestinal tract without causing problems. In the majority of cases, the patients do not recall the act or time of foreign body ingestion. ${ }^{[6]}$ There are several case reports in the literature regarding perforations caused by toothpicks, fish bones, chicken bones, needles, pins, wood splinters, seeds, parasites, tomato skin, a button battery, and an alkaline battery ${ }^{[8,9]}$. There seems to be a tendency for foreign bodies to lodge in the blind pouch of Meckel's diverticulum. Back in 1950, Ward-McGuard described the mechanism of perforation as a combination of local inflammation due to irritation of the foreign body, and pressure necrosis of the divertculum wall, secondary to attempts by peristalsis to push the foreign body toward the tip of the diverticulum. ${ }^{[10]}$

A preoperative diagnosis of acomplicated Meckel's diverticulum may be challenging because of the overlapping clinical and imaging features of other acute surgical and inflammatory conditions of the abdomen. Efficiency of diagnostic imaging varies with this disease process. Plain films are usually nonspecific. Radionuclide scintigraphy will detect $85 \%$ of Meckel's cases if ectopic gastric mucosa is present in the diverticulum. Enteroclysis may also detect a smaller percentage of diverticula, ranging up to $75 \%$.Abdominal CT may yield a high rate of diagnosis when small bowel obstruction is present $(81 \%$ to $96 \%)$, but a Meckel's etiology is difficult to identify as a cause due to the inability to distinguish a diverticulum amongst loops of small bowel. ${ }^{[12]}$

In a 2005 retrospective study, Ueberrueck et al. analyzed the significance of MD in cases diagnosed as appendicitis. In a 26-year period, a total of approximately 10000 appendectomies were performed. The bowel was explored to search for a MD in approximately $80 \%$ of these cases. The presence of a Meckel's was discovered in $3 \%$ of these cases, while $9 \%$ of these diverticulums were found to have pathology, including obstruction, diverticulitis, perforation, and intussusception. This study concluded in establishing the importance of exploring the bowel in all appendectomy cases. $^{[13]}$

In patients with the preoperative workup that raises suspicion for, but without an intraoperative verification of, an acute appendicitis, an exploration of $180 \mathrm{~cm}$ of the small bowel from the ileocecal valve is advocated. Both pathologies being present, is very rare, and therefore little is to be gained by searching for a diverticulum where acute appendicitis is present and dealt with. However, some authors recommended that Meckel's diverticulum should be looked for in all cases of appendicitis and if found, it should be removed. ${ }^{[14]}$

In 2005 Mayo, retrospective, 52-year period study including 1476 patients, authors analysed which diverticulum should be removed when discovered incidentally during abdominal surgery. Study included data such as preoperative diagnosis, age, sex, date of surgery, and intraoperative, macroscopic, and microscopic findings from operative and pathology reports, and then, usinglogistic regression analysis, determined which clinical or histologic features were associated with symptomatic MD. The conclusion was that if patient fulfills one of the four criteria: age younger than 50 years; male sex;diverticulum length greater than $2 \mathrm{~cm}$; and if there is presence of histologically abnormal tissue - that diverticulum should be removed. ${ }^{[15]}$ 
Regardless of the fact that a variety of diagnostic procedures are at the disposal of surgeons, we feel that the diagnose of a complicated MD remains a challenging task, largely in thanks to the fact that it mimics some other acute abdominal pathology and, often, is misdiagnosed.

\section{References}

1. Sagar J, Kumar V, Shah DK. Meckel's diverticulum: A systematic review. J R Soc Med 2007 Feb; $100(2): 69$.

2. Kloss B.T., Broton C.E., Sullivan A.M. Perforated meckel diverticulum. Int J Emerg Med (2010) 3: 455-457.

3. Dumper J, Mackenzie S, Mitchell P, et al. Complications of Meckel's diverticula in adults. Can J Surg. 2006 Oct; 49(5): 353-357.

4. Di Saverio, et al. WSES Jerusalem guidelines for diagnosis and treatment of acute appendicitis. World Journal of Emergency Surgery 2016; 11: 34 .

5. Sagar J, Kumar V, Shah DK. Meckel's diverticulum: a systematic review. J R Soc Med 2006 Oct; 99(10): 501-505.

6. Dimitriou I, et al. Perforation of Meckel's diverticulum by a fishbone presenting as acute appendicitis: A casereport. Journal of Medical Case Reports 2013; 7: 231.

7. Aamery A, Al-Shehhi R, Malik K, et al. Perforation of Meckel's diverticulum with a foreign body mimicking acute appendicitis: A rare complication. J Pak Med Assoc 2017 Jun; 67(6): 942-944.

8. Roosswick RP: Perforation of Meckel's diverticulum by foreign bodies. Postgraduate Medical J 1965; 41: $105-106$.

9. Willis GA, Ho WC: Perforation of Meckel's diverticulum by an alkalinehearing aid battery. CMAJ 1982; 126: 497-498.

10. WVard-McGuard, N. N.: Perforated Meckel's Diverticulum by Tomato Skin. Lancet, 1: 349, 1950.

11. Malik AA, Shams-ul-Bari, Wani KA, et al. Meckel's diverticulum - revisited. Saudi J Gastroenterol 2010; 16(1): 3-7.

12. Murakami R, Sugizaki K, Kobayashi Y, et al. Strangulation of small bowel due to Meckel diverticulum: CT findings. Clin Imaging 1999; 23: 181-183.

13. Ueberrueck T, Meyer L, Koch A, et al. The significance of Meckel's diverticulum in appendicitis-a retrospective analysis of 233 cases. World J Surg 2005; 29: 455-458.

14. Matsagas MI. Incidence, complications and management of Meckels Diverticulum. Arch Surg 2005; 130 : $143-6$.

15. Park JJ, et al. Meckel diverticulum: The mayo clinic experience with 1476 patients (1950-2002). Ann Surg 2005 Mar; 241(3): 529-33. 